\title{
Aspects aléatoires de la dynamique de la différenciation cellulaire
}

Pour le profane comme pour le spécialiste, le développement d'un organisme vivant est une source d'émerveillement intarissable. La succession des générations cellulaires qui, partant d'une unique cellule œuf, conduisent à un organisme doté de tissus et d'organes aux fonctions variées fascine par émergence de la complexité.

Même si elle ne peut être abordée de front, la recherche de ce qui, dès la cellule initiale, est déterminé, et de la forme physique de ce déterminisme, est centrale en embryologie. Contrairement à d'autres branches de la biologie, comme la physiologie par exemple, dans lesquelles le milieu extérieur et ses variations jouent un rôle déterminant, il se dégage de l'observation de l'ontogenèse des métazoaires une impression de très grande autonomie car les principaux moments de la vie embryonnaire comme la segmentation de l'œuf, la gastrulation, la neurulation, etc., ont une chronologie si bien établie que la seule indication de l'âge de l'embryon à partir de la fécondation permet de préciser son stade de développement. La découverte des gènes homéotiques ou gènes du complexe Hox, dont l'expression structure l'embryon en positionnant les cellules les unes par rapport aux autres, est venue renforcer cette impression de très forte détermination en lui donnant la base moléculaire qui lui manquait encore $\left(m / s n^{\circ} 2\right.$, vol. 10, p. 145). Le concept si populaire de "programme génétique" a donc trouvé la preuve irréfutable de son bien-fondé lorsque les étapes les plus précoces du développement ont elles-mêmes trouvé leurs gènes. Le développe- ment semble donc pouvoir se résumer à une grande cascade de gènes s'appelant les uns les autres comme se succèdent les différentes instructions d'un programme informatique. Pourtant, un certain nombre de publications récentes fait état de phénomènes de différenciation cellulaire aléatoires. Une cellule souche paraît pouvoir s'engager dans telle ou telle autre voie de différenciation au hasard. Ces observations ont été faites sur des organismes divers et sur plusieurs types de tissus. Les exemples sont maintenant suffisamment nombreux pour ne pas se réduire à de simples curiosités exotiques. Ce que nous savons du développement des métazoaires et la manière dont nous le comprenons vont tout à fait à l'encontre de ce genre d'observations qui sont même un peu troublantes tant elles cadrent mal avec les schémas explicatifs actuels.

Cette revue rappellera quelques-uns des exemples de différenciation aléatoire connus aujourd'hui. Elle cherchera ensuite à discuter la mesure dans laquelle ces phénomènes aléatoires peuvent être réintégrés à notre compréhension globale du développement. Mais avant de s'engager sur le terrain incertain des phénomènes aléatoires, quelques brefs rappels mathématiques et épistémologiques sur le sens d'une modélisation stochastique aideront sans doute à aborder le thème avec une plus grande sérénité.

\section{Phénomènes aléatoires}

La notion de hasard, de phénomène aléatoire ou encore stochastique (stochastique étant synonyme d'aléa- toire) a considérablement évolué au cours des quarante dernières années. Sans chercher à engager un grand débat philosophique, il peut être utile de rappeler brièvement les éléments du problème [1]. Le hasard est un concept flou qu'il semble préférable d'éliminer du discours. En revanche, il est primordial de clarifier le sens et les justifications possibles d'un recours à des modèles stochastiques.

Fondamentalement, la seule notion non ambiguë est mathématique ; c'est celle de probabilité qui permet de conduire des calculs sur des ensembles d'événements en se fondant sur quelques axiomes directement issus de l'expérience quotidienne. Plus un événement "a de chances" d'être réalisé, plus sa probabilité est proche de 1 . Un événement certain a une probabilité 1 et un événement impossible a une probabilité 0 . Toujours, la somme des probabilités de tous les événements possibles est égale à 1 . Les probabilités sont la branche des mathématiques qui permet de traiter les situations où plusieurs possibilités simultanées coexistent. Une branche des probabilités, la théorie des processus stochastiques, traite les cas d'évolutions temporelles des systèmes probabilistes. A chaque instant, ces systèmes peuvent changer d'état avec une composante aléatoire dans le changement d'état. Ainsi, la connaissance de l'état du système à un instant donné ne permet pas de prédire dans quel état il se trouvera à un instant ultérieur. Cette prédiction ne pourra se faire qu'en probabilité. Les modèles mathématiques déterministes sont plus généralement utilisés dans l'étude des phénomènes 
dynamiques, alors que les équations différentielles, permettent, au contraire, ce genre de prédictions. Il faut souligner l'analogie très forte qui existe entre les systèmes dynamiques déterministes et les systèmes dynamiques aléatoires. En effet, l'équation qui régit l'évolution d'un système déterministe peut être représentée de la manière suivante:

$$
\overrightarrow{X_{t}}=A \vec{X}_{0}
$$

expression dans laquelle A représente un opérateur qui établit une relation entre l'état initial et l'état au temps t. Pour un processus aléatoire, cette relation prend exactement la même forme, mais au lieu de porter sur l'état du système, elle affecte tous les états possibles du système d'une probabilité qui dépend du temps. De là, il découle qu'une évolution déterministe n'est qu'un cas particulier d'évolution aléatoire, c'est une évolution de probabilité 1 . Cette remarque qui peut sembler un peu gratuite a son importance. Elle signifie que la dichotomie entre aléatoire et déterministe souvent tenue pour irréductible n'a, mathématiquement au moins, pas lieu d'être.

Si mathématiquement tout est limpide, il n'en est pas de même en sciences expérimentales où l'emploi d'un modèle aléatoire est bien souvent justifié par "l'intervention du hasard " dans le phénomène observé. Ce prétendu hasard empoisonne un grand nombre de discours parascientifiques. Afin de pouvoir poursuivre l'analyse, il convient de rappeler que cet agent extérieur n'a sa place dans aucune démarche scientifique car il traduit souvent une pensée finaliste ou anthropomorphique. La prise en compte de phénomènes aléatoires ne consiste pas à étudier l'influence du doigt de Dieu dans l'évolution de l'objet étudié !

Classiquement, les modèles probabilistes ont été utilisés pour faire face aux situations d'ignorance reconnue. En théorie des jeux, par exemple, il est nécessaire de faire appel à un raisonnement probabiliste car les revers des cartes étant tous identiques, chaque joueur se trouve en situation d'ignorance des jeux de ment que d'une connaissance globale de la composition du jeu qui s'affine levée après levée. En génétique quantitative, l'emploi d'un modèle probabiliste découle $\mathrm{d} u$ fait que les caractères étudiés sont l'expression conjointe d'un grand nombre de gènes non identifiés et d'un milieu physique non mesurable à l'échelle d'un organisme entier et dont les relations avec lui ne sont pas parfaitement élucidées, etc. On pourrait multiplier les exemples. Cette situation d'ignorance doit être levée et une description plus minutieuse de l'objet étudié devrait, dans ces situations, permettre de dépasser le modèle probabiliste.

Les possibilités de simulations numériques offertes par l'informatique sont venues bouleverser un peu cette conception classique de l'aléatoire. On s'est en effet rendu compte qu'une certaine classe de modèles déterministes présentait une grande sensibilité aux conditions initiales, ce qui se traduit, en pratique, par le fait que les évolutions de deux états initiaux très proches du système divergent rapidement de manière significative. La science du XIX ${ }^{e}$ siècle, celle qui est enseignée à l'école et dans les premières années d'université, nous a façonné l'esprit avec des modèles qui, au contraire, ont la sympathique propriété d'assurer à deux points voisins, des évolutions similaires. Une bonne part de la capacité prédictive de la physique provient de cette particularité. Physiquement, les systèmes modélisables par des équations présentant une grande sensibilité aux conditions initiales doivent souvent être abordés avec des outils probabilistes du simple fait que l'observation de leur état est toujours entachée d'une erreur de mesure qui est un obstacle insurmontable à la prédiction. James Gleick a donné une introduction très vivante à ces questions [2] . Cette révélation assez récente signifie que l'étude d'un phénomène physique avec des méthodes probabilistes n'en dénonce pas forcément une description encore sommaire. Le biologiste, en revanche, généralement peu familiarisé avec les notions de sensibilité aux conditions initiales et d'instabilité dynamique, tend sou- vent à chercher dans une dissection plus fine encore le détail qui permettrait de mettre en évidence un comportement plus simple.

Ainsi donc, aussi bien mathématiquement que physiquement, il n'y a pas d'alternative irréductible entre une approche probabiliste et une autre déterministe. Il n'y a qu'une palette de représentations possibles d'un phénomène physique au sein de laquelle la plus adaptée doit être choisie. Le critère de choix reposera largement sur le type d'observations disponibles et surtout sur la complexité de la dynamique du phénomène.

\section{Hématopoïèse}

Il ne s'agit pas ici de dresser un catalogue de tous les phénomènes aléatoires rencontrés en biologie du développement. Une telle revue ne pourrait être dressée que par un auteur ayant une connaissance très approfondie et très large de l'embryologie. En revanche, on se propose de rappeler quelques exemples dans lesquels l'apparition d'un type cellulaire nouveau est régie par un processus aléatoire. C'est donc sur la transformation qualitative que subit l'embryon à cette occasion que sera centrée la suite du texte et non sur d'éventuelles fluctuations quantitatives. Certains exemples ont été décrits récemment et d'autres sont plus anciens. L'objectif est de soulever le problème qu'ils posent globalement. La présentation des résultats insistera sur les méthodes expérimentales ayant permis de démontrer ou de soupçonner le caractère aléatoire des phénomènes. L'hématopoïèse est un champ particulièrement fertile en différenciations aléatoires mais non exclusif.

\section{- Les travaux de Till}

L'importance de phénomènes aléatoires dans certaines étapes de l'hématopoìèse a été entrevue dès 1964 [3]. Les moyens d'observation étaient alors beaucoup plus rudimentaires et la description des populations cellulaires beaucoup moins fine qu'aujourd'hui. Till et al. distinguent trois populations correspon- 
dant à trois stades de développement : les cellules souches non différenciées, qui ont une grande aptitude à la prolifération; elles peuvent se multiplier ou s'engager dans une voie de différenciation; elles rejoignent alors les cellules peu différenciées qui ont une aptitude à la prolifération plus limitée ; enfin, on trouve les cellules spécialisées, qui ont perdu toute capacité de prolifération. Ce sont les cellules de ce der- nier groupe qui sont fonctionnelles. Il avait été observé que la taille de cette population cellulaire est relativement constante en conditions normales mais peut rapidement augmenter en cas de stress. Le nombre de cellules fonctionnelles était donc soumis à un contrôle assez fin. Un modèle hormonal avait été proposé, mais Till et al. ont exploré une autre voie.

A l'époque, aucun marqueur parti-

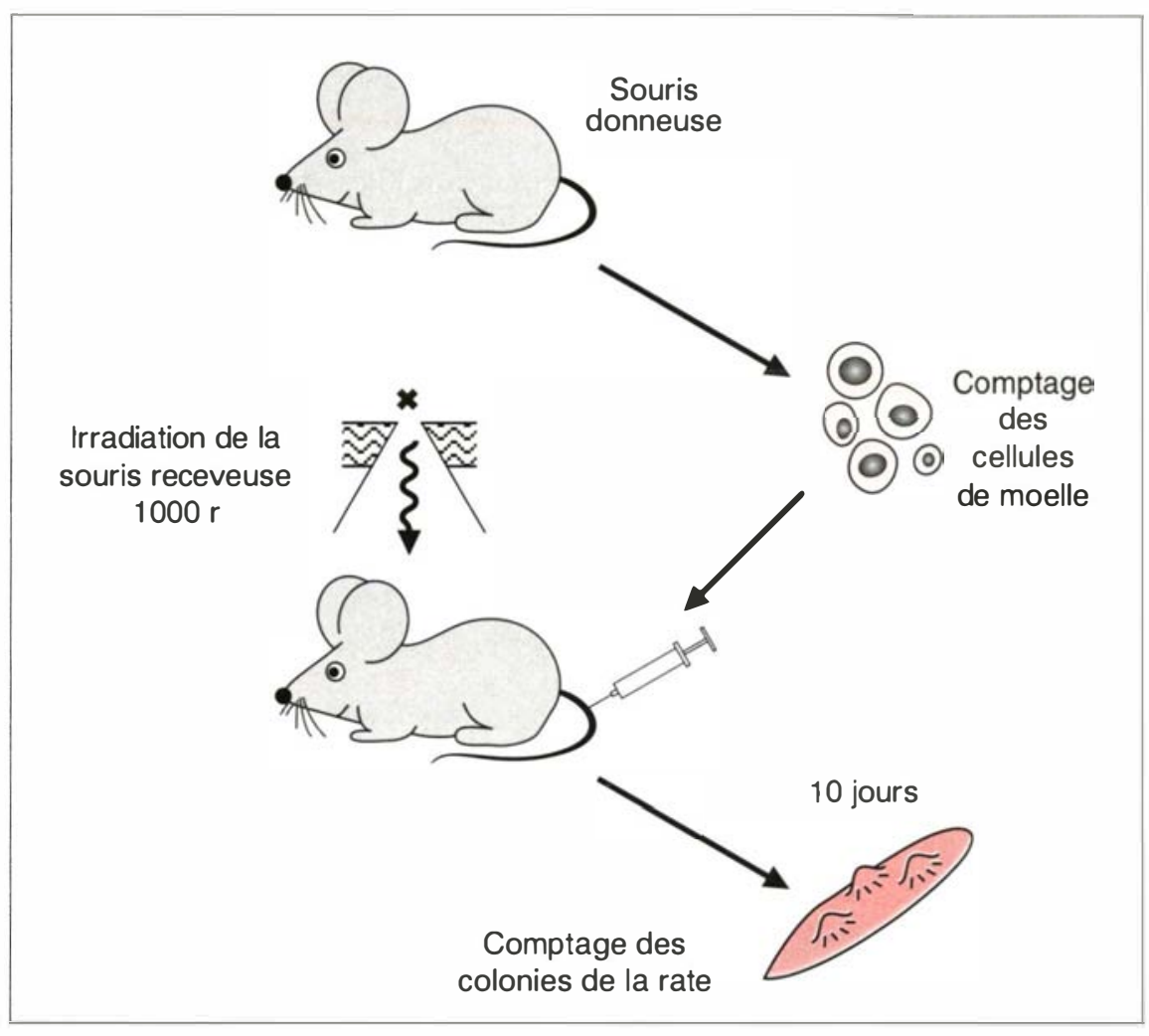

Figure 1. Les expériences de Till. La procédure peut être résumée ainsi. Une suspension de cellules de moelle osseuse est injectée, après comptage, à des souris ayant subi une irradiation intense. Après dix jours, les animaux sont tués et leur rate prélevée. Les colonies apparaissent sous la forme de nodules bien isolés et faciles à compter; elles sont issues de la division d'une unique CFC (colony forming cell). Cette CFC est caractérisée par l'aptitude des cellules de la colonie qu'elle a formée à donner, elles-mêmes, des colonies. La colonie est prélevée, dispersée et injectée à son tour à des souris irradiées sur lesquelles on mesure 10 jours plus tard le nombre de colonies appelé unité de formation de colonie (CFU). Un coefficient correcteur, tenant compte du fait que toutes les cellules injectées n'atteignent pas la rate, est appliqué à ce nombre de colonies pour obtenir le nombre de cellules capables de former des colonies présentes dans la suspension injectée. Cette expérience a été conduite sur 89 colonies. L'histogramme des fréquences présente une variance importante et un bon ajustement à une loi Gamma. Les fluctuations de l'échantillonnage ne peuvent, à elles seules, rendre compte de cette distribution des résultats. (D'après [3].) culier des cellules souches n'avait été identifié mais un test biologique avait été décrit. Une greffe de moelle osseuse est réalisée sur une souris irradiée. Dix jours plus tard, les rates des souris greffées sont prélevées et l'on observe des colonies d'environ $10^{6}$ cellules issues chacune d'une seule cellule notée CFC (colony forming cell). L.es colonies comportent, d'une part, des cellules différenciées et, d'autre part, des CFC puisque les cellules de colonies injectées à des souris irradiées forment à leur tour des colonies. Cet ensemble de caractéristiques laisse penser que les CFC sont "proches " des cellules souches; elles forment peut-être une sous population des cellules souches (figure 1).

Le nombre de CFC issues d'une colonie est noté CFU (colony forming unit) et peut être considéré comme une caractéristique de la cellule souche ayant fondé la colonie. Si le contrôle de la différenciation et de la prolifération des cellules souches est très strict, on peut s'attendre à ce que les CFU des cellules souches d'un même prélèvement de moelle soient proches. Au contraire, si des phénomènes aléatoires interviennent alors, de grandes variations seront observées. Et c'est bien le résultat de l'expérience. Pour un seul prélèvement de moelle initial, le nombre de CFU a un écart-type très supérieur à la moyenne et se répartit selon une loi de probabilité Gamma dont les erreurs d'échantillonnage ne suffisent pas à rendre compte. Un autre phénomène entre donc en jeu. Chaque CFC a une probabilité $\mathrm{p}$ de s'engager dans une voie de différenciation qui la prive de son aptitude à former des colonies et une probabilité (1-p) de se diviser en deux CFC, ce type de comportement correspond à un modèle très classique de la théorie des processus stochastiques nommé processus de naissance et de mort. Des simulations ont montré que les données expérimentales obtenues étaient compatibles avec les lois de probabilité obtenues par ces processus. 
- Éducation des cellules T

La différenciation des lymphocytes $\mathrm{T}$ offre encore un exemple de phénomène de différenciation aléatoire d'autant plus intéressant que l'aspect aléatoire du phénomène n'a été reconnu que tardivement et reste d'ailleurs très controversé [4]. Il existe deux classes de lymphocytes $\mathrm{T}$ que les marqueurs membranaires CD4 et CD8 permettent de distinguer. Les lymphocytes $\mathrm{T}$ $\mathrm{CD} 4^{+} \mathrm{CD} 8$ ont un récepteur (TCR) capable de reconnaître l'antigène présenté par des molécules d'histocompatibilité de classe II. Ils permettent, entre autres, la prolifération des lymphocytes B; ce sont les lymphocytes $\mathrm{T}$ auxiliaires. Pour leur part, les lymphocytes T CD4-CD8+ ont un récepteur capable de reconnaître des peptides antigéniques dans le contexte d'une molécule d'histocompatibilité de classe I. Ils ont une activité cytotoxique. De cette typologie se dégage une relation forte entre la spécificité du récepteur et la présence à la surface de la cellule de l'un ou l'autre des deux marqueurs. De plus, il faut savoir que les précurseurs de ces deux types de cellules sont des thymocytes qui expriment à leur surface les deux marqueurs CD4 et CD8, ils sont dits doubles positifs (DP).

Deux modèles explicatifs ont été proposés (figure 2). Le premier est déterministe : c'est le modèle de l'instruction qui postule que c'est l'interaction entre le récepteur $\mathrm{T}$ et la molécule présentatrice de l'antigène qui a le premier rôle. Seule la molécule qui est coengagée dans l'interaction TCR-CMH voit son expression maintenue alors que celle qui ne peut être engagée dans cette interaction disparaît. C'est donc l'interaction TCR-CMH sur le thymocyte qui instruit le marqueur et la fonction de la future cellule $\mathrm{T}$. L'autre modèle est probabiliste: c'est le modèle de la différenciation par sélection. On suppose ici que l'expression de l'un ou l'autre des deux marqueurs s'arrête de manière aléatoire, puis que, dans une deuxième étape, seules les cellules fonctionnelles sont sélectionnées et prolifèrent. Les cellules fonction-

\section{A. Instruction déterministe}
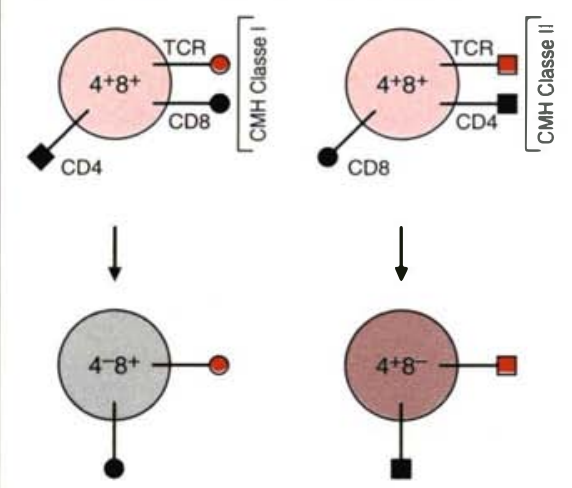

\section{B· Sélection aléatoire}
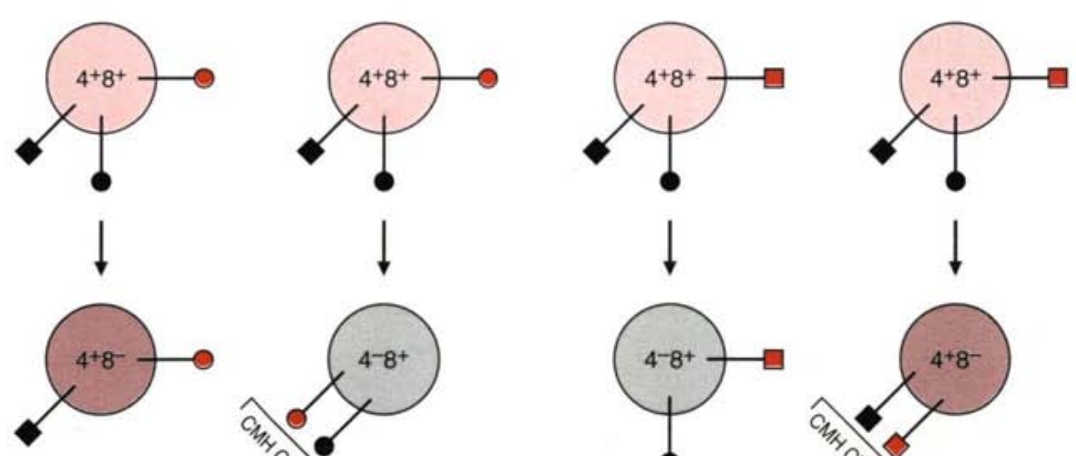

$t$

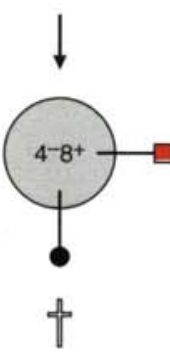

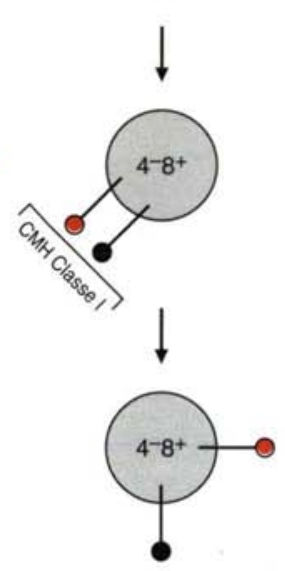

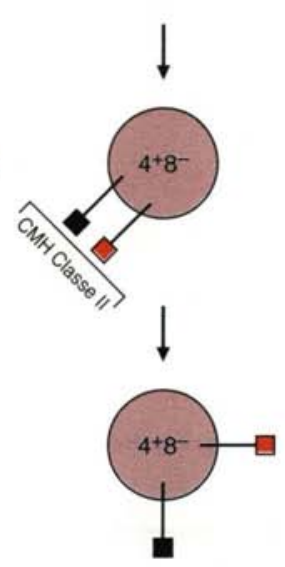

Figure 2. Deux modèles de l'engagement des cellules dans les voies CD4 et CD8. Les cellules T immatures ont, soit un récepteur (TCR) spécifique d'une molécule d'histocompatibilité de classe I (disque rouge), soit d'une molécule de classe II (carré rouge), et présentent à leur surface les deux co-récepteurs CD4 (carré noir) et CD8 (disque noir). Dans le modèle de l'instruction (A), la formation du complexe CD4-TCR-CMH II induit un signal qui provoque la disparition du deuxième corécepteur CD8. D'une manière similaire, la formation du complexe CD8-TCR-CMH I conduit au phénotype CD8+CD4. Dans le modèle stochastique de la sélection (B), l'expression de l'un des deux corécepteurs est perdue par les cellules doubles positives d'une manière aléatoire indépendante de la spécificité du TCR. Dans une deuxième étape de sélection, les cellules fonctionnelles, celles dont le corécepteur est compatible avec le récepteur, engagent une interaction avec une molécule du $\mathrm{CMH}$. Cette interaction les maintient en vie. Les cellules non fonctionnelles ne peuvent pas engager cette interaction et meurent. (D'après [4].) 
nelles sont celles pour lesquelles les spécificités du récepteur et du marqueur membranaire sont cohérentes.

L'étude des populations de thymocytes permet de distinguer les deux modèles. I.e modèle aléatoire implique qu'une population de cellules immatures avec un récepteur et un marqueur incohérent (C.D4 avec un récepteur spécifique des molécules de classe I et CD8 avec des molécules de classe II) doit exister. Aucun des deux modèles ne s'est encore imposé [4].

U'ne première série d'expériences laissait penser que le modèle de l'instruction était le plus probable. Ainsi, l'expression de (.D8 sur tous les lymphocytes $T$ immatures et mûrs dans des souris transgéniques exprimant aussi un TCR transgénique spécifique des molécules du CMH de classe I ne permet pas d'observer la récupération de cellules $\mathrm{CD}^{+}[5]$. De même, l'expression d'un corécepteur chimérique formé de la queue cytoplasmique de CD4 et de la partie extracellulaire de CD8 conduit à l'obtention d'une population de lymphocytes T (:D4 ${ }^{+}$ exprimant un TC:R spécifique des molécules du C.MH de classe I [6].

Des résultats plus récents sont venus remettre en cause cette idée. Ainsi, la surexpression transgénique de CD4 dans des souris dépourvues de molécules de classe I permet d'identifier une population de cellules qui expriment CD8 mais qui n'expriment pas le CD4 endogène. La molécule C.D4 transgénique permet la sélection positive des cellules CD8 ${ }^{+}$exprimant pourtant un TC:R spécifique de la classe II [7]. Plus récemment et venant contredire les résultats précédemment cités [5], l'utilisation de souris transgéniques pour C.D8 et pour un TCR (différent du précédent) restreint aux molécules de classe I a permis de mettre en évidence une population CD4 ${ }^{+} \mathrm{TCR}^{+}$[8]. Enfin, il a aussi été montré sur des souris n'exprimant pas de produits du C.MH de classe II qu'il existe une population significative de cellules C.D4 $4^{+}$.D8 . ( es cellules présentent des caractères de maturité intermédiaires entre les précurseurs doubles positifs et les simples positifs en fin de différenciation. De façon similaire, il existe une population de simples positifs intermédiaires CD4C.D8 ${ }^{+}$chez des souris n'exprimant pas de produit du C:MH de classe I [9].

La récente description d'une nouvelle population de lymphocytes $T$ présentant un phénotype original (CD 4+CD8 ou CD4-CD8 ) et une réactivité spécifique de la classe I est venue quelque peu ébranler la vue générale de l'éducation thymique [10]. Il semble trop tôt aujourd hui pour domner, des expériences précédemment citées, une interprétation cohérente avec ces nouvelles données. Une chose est cependant certaine, le sujet n'est pas clos.

Il convient de faire preuve de la plus grande prudence dans l'interprétation de résultats de ce type, comme le montre l'exemple suivant. Des expériences mettant en évidence qu'une unique cellule souche de la moelle osseuse pouvait engenclrer, d'une part, les tissus mésenchymateux de l'envirommement de l'hématopoïèse et, d'autre part, les cellules souches de l'hématopoïèse ont été publiées en 1992 [11]. L'engagement dans l'une des deux voies était alors supposé aléatoire. Il s'est en fait avéré que ces résultats n'ont jamais pu être reproduits et que, par conséquent, les conclusions de l'article étaient injustifiées [12].

\section{Différenciation des crêtes neurales}

L.e fait que les précédents exemples relèvent tous de l'immunologie ne doit pas laisser penser que les phénomènes de différenciation aléatoires se cantomnent à ce domaine. D'autres exemples ont été décrits. Comme les travaux de Novak et Till, ceux conduits par Baroffio et Blot font appel aux techniques d'analyses statistiques appropriées pour mettre en évidence le caractère stochastique de certaines étapes de la différenciation des cellules céphaliques des crêtes neurales (cellules NC) d'embryons de cailles [13].

Les cellules NC: sont pluripotentes car, mises en culture sur un tapis de fibroblastes 3T3 dans un milieu homogène adapté, elles se différencient en cinq principaux types cellu- laires qui sont les neurones, les cellules adrénergiques, les cellules de Schwann, les mélanocytes et les chondrocytes. Lorsque les cellules NC: sont clonées, elles forment des colonies de tailles variables dans lesquelles on trouve un à cinq des types cellulaires ci-dessus et toutes les associations possibles de types cellulaires au sein d'une colonie semblent pouvoir apparaître. Si l'engagement d'une cellule dans une voie de différenciation est aléatoire et indépendant de son environnement, alors aucune corrélation particulière ne doit être observée entre les différents caractères des colonies issues du clonage des cellules NC: (présence ou absence d'un type cellulaire au sein de la colonie). I.es 201 clones de cellules NC: qui ont été étudiés ont été décrits au moyen de sept variables phénotvpiques. L'étude d'un tel ensemble de domnées nécessite une méthodologie appropriée. U'n premier calcul, appelé " analyse factorielle des correspondances ", foumit des indications purement descriptives sur les variables corrélées et les variables indépendantes au sein de l'échantillon étudié. Une deuxième étape permet l'interprétation des résultats de la première; pour chaque couple de caractères, on estime la probabilité que les deux caractères soient indépendants au moyen d'un test d'ajustement. La principale conclusion de l'étude est que les présences de cellules de Schwann, de cellules adrénergiques et de neurones au sein d'un clone sont fortement corrélées. En revanche, les différenciations en cellules pigmentées et en cartilage sont indépendantes l'une de l'autre et sont chacune indépendantes de la différenciation en chacun des trois types cellulaires associés ci-dessus. Afin de démontrer la généralité de la problématique, citons pour mémoire un dernier exemple, le développement des gonades du ver C. elegans (figure 3): deux cellules Zl.ppp et Z4.aaa forment un groupe d'équivalence (groupe de cellules ayant les mêmes potentialités). En l'absence de contacts cellulaires, elles s'engagent dans la voie AC. (anchor cell) qui provoquera ultérieu- 
rement l'induction de la vulve. Au cours du développement normal de l'embryon, la situation est différente puisque l'une des deux s'engagera dans la voie de différenciation AC tandis que l'autre ira dans la voie VU (ventral uterine) [14]. L'intérêt de cet exemple est que des études génétiques ont permis de proposer des modèles d'interaction entre les deux cellules rendant compte de la différenciation.

\section{Aux limites de l'observation}

La mise en rapport de quelques remarques générales sur les phénomènes aléatoires et de quelques exemples de différenciation cellulaire aléatoire n'est pas innocente. Elle voudrait promouvoir l'idée qu'un comportement aléatoire à l'échelle cellulaire n'est pas forcément pathologique et mérite au contraire d'être considéré systématiquement avec les méthodes adéquates.

L'emploi d'une représentation stochastique suscite souvent une certaine réticence que l'on peut attribuer à une confusion trop fréquente entre aléatoire et anarchique. Or, il faut rappeler qu'un phénomène aléatoire n'est pas nécessairement complètement désordonné (le désordre maximal n'est obtenu que pour les phénomènes distribués selon une loi uniforme). Certains des exemples mentionnés ci-dessus présentent au contraire une très grande régularité de comportement. Ainsi l'engagement dans les voies VU ou AC est exclusif de toute autre issue et chacune de ces deux voies donne un embryon " correctement " formé. Cet exemple correspond parfaitement à un phénomène aléatoire puisqu'un unique état initial (l'appartenance à un même groupe d'équivalence) conduit à deux états distincts. Le plus étonnant est qu'à l'échelle de l'organisme, chacune de ces deux routes conduit au même état, un embryon normal.

Les bases moléculaires des phénomènes de différenciation rappelés ci-dessus restent dans l'ensemble largement ignorées, même si quelques revanche, il est difficile de ne pas rapprocher ces exemples du plus simple des développements, celui du bactériophage lambda. On considère en effet que les deux voies, lytique et lysogénique, empruntées par le phage au moment de l'infection, constituent en quelque sorte la version minimale d'une étape de développement. De plus, le "choix" entre chacune des voies est aléatoire et la probabilité d'emprunter chacune des voies dépend des conditions physiologiques de l'hôte [15]. Lors de l'infection par le phage lambda, un unique état initial peut conduire à deux états asymptotiques très différents. L'un, la lysogénie, est stable tandis que l'autre, la lyse, est explosif (au sens mathématique). L'instabilité initiale résulte de deux caractéristiques. D'une part, les protéines $\mathrm{CI}$ et Cro ont des effets régulateurs opposés et, d'autre part, elles sont présentes initialement à très faible concentration, ce qui provoque d'importantes fluctuations. En effet, une variation de quelques molécules par cellule provoque une variation importante $d u$ rapport des quantités de CI à celles de Cro. Ces fluctuations sont probablement d'autant plus importantes qu'il semble bien que la transcription possède, elle-même, un certain caractère aléatoire lié, lui aussi, au faible nombre des molécules en jeu [16-18]. On peut regretter que ces questions n'aient pas encore été abordées systématiquement. Tous les travaux de modélisation de l'infection sur la base des données thermodynamiques disponibles se contentent d'étudier la stabilité des états stationnaires [19, 20], mais aucune étude dynamique ne semble avoir encore été entreprise. Et c'est pourtant cet aspect dynamique qui paraît biologiquement le plus pertinent. Il est raisonnable de penser que ces caractéristiques de l'infection par le phage lambda présentent un certain degré d'universalité dans la biologie du développement [15] et l'analogie, par exemple, avec le modèle proposé pour la décision VU-AC chez C. elegans est très frappante.

Les exemples biologiques mentionnés ici voudraient illustrer le lien

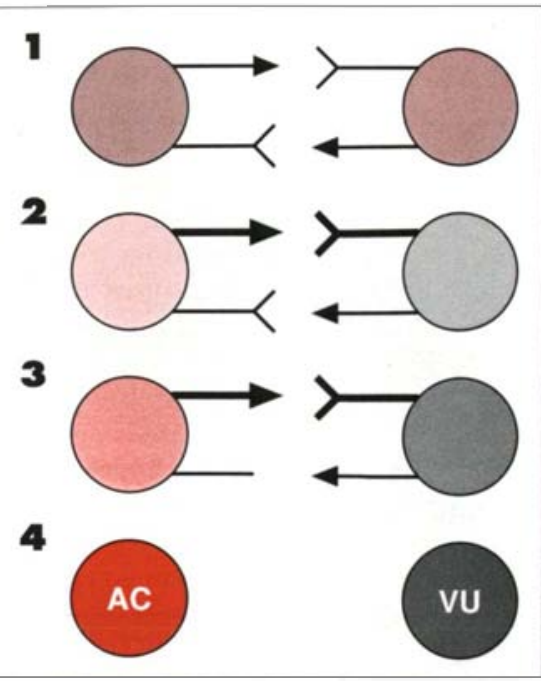

Figure 3. Modèle de l'engagement dans les voies $A C$ et $V U$ des cellules Z4.ppp et Z4.aaa. C'est l'interaction entre les deux cellules qui spécifie leur devenir respectif. Étape 1: Les deux cellules sont équivalentes, ce qui implique qu'elles ont la même activité d'émission (flèche) et de réception (flèche inversée) du signal. La nature du signal est inconnue mais son récepteur est probablement le produit du gène Lin-12. Étape 2: Des fluctuations aléatoires créent un solde non nul d'émission en faveur de l'une des deux cellules qui devient globalement émettrice alors que l'autre devient réceptrice. Étape 3 : L'activité émettrice de la cellule réceptrice est réduite alors que son activité réceptrice Lin-12 est au contraire stimulée. Étape 4: L'engagement de chacune des cellules dans sa propre voie est maintenant irréversible et les activités d'émission et de réception de signal ne sont plus nécessaires. (D'après [14].) 
très fort qui unit l'apparence stochastique d'un phénomène et la mesure qui le décrit. Dans le cas de la différenciation cellulaire, les mesures doivent porter sur des cellules prises individuellement et dans un état initial égal au regard de la mesure. L'originalité des phénomènes aléatoires réside dans le fait qu'un même état initial peut conduire à plusieurs états ultérieurs différents. Pour les mettre en évidence, il faut donc être en mesure de suivre l'évolution de plusieurs individus placés dans un même état initial. La mesure ne peut donc être qu'individuelle. De plus, un même état initial correspond, en fait, à un ensemble d'états indistinguables avec les moyens d'observation mis en ouvre. Cette remarque permet une relecture des différents exemples proposés. Dans le cas des travaux de Till, des cellules NC ou de la décision VU-AC, les mesures sont bien effectuées sur des cellules individuelles et le caractère aléatoire des observations est donc rigoureusement établi. En revanche, aucun des travaux relatifs à la différenciation des cellules $T$ ne repose sur une observation individuelle des précurseurs des cellules T. Seules des populations cellulaires ont été observées et le caractère aléatoire de l'enchaînement des événements n'est qu'une interprétation qui permet d'unifier, au sein d'un modèle cohérent, les différentes mesures qui ont été faites de ces populations. Il demeure essentiellement spéculatif.

Comme le caractère aléatoire de la différenciation est intrinsèquement lié aux mesures permettant d'observer cette différenciation, il n'est pas nécessairement définitif. L'arrivée de nouvelles techniques d'observation peut, à tout moment, le remettre en cause. L'étude de la différenciation des cellules des crêtes neurales repose sur l'apparition de colonies lors de leur mise en culture. L'observation de ces colonies ne prend en compte que des critères phénotypiques des cellules qui les composent mais d'autres observations, telles que les positions de chacune des cellules dans les colonies, ne sont, en revanche, pas prises en compte. Or, l'étude des phéno- mènes d'induction à l'échelle de l'embryon montre clairement que l'organisation spatiale est capitale dans le développement. Les cellules, du fait de leur répartition dans l'espace, s'influencent mutuellement $[14,21]$. Demain, la prise en compte de mesures topographiques, ou de toute autre mesure, dans l'étude de ces phénomènes permettra peutêtre de dégager de nouveaux déterminismes et l'aléa d'aujourd'hui s'effacera

\section{Remerciements}

Jean Peccoud exprime ici toute sa gratitude à Jacques Demongeot pour les nombreuses conversations qui sont à l'origine de ce travail qui n'aurait pas été possible sans le soutien financier de la Fondation pour la recherche médicale. Stéphane Viville pour sa part tient à remercier l'EMBO pour son soutien financier.

\section{RÉFÉRENCES}

1. Amsterdamski S, Atlan H, Danchin A, et al. La querelle du déterminisme. Paris: Gallimard, 1990.

2. Gleick I. La théorie du chaos. Paris : Albin Michel, 1989.

3. Till JE, McCulloch EA, Siminovitch L. A stochastic model of stem cell proliferation, based on the growth of spleen colonyforming cells. Proc Natl Acad Sci USA 1964; $61: 29-36$

4. von Boehmer H, Kisielow P. Lymphocyte lineage commitment: instruction versus selection (comment). Cell $1993 ; 73: 207-8$.

5. Borgulya P, Kishi H, Muller U, Kirberg J von Boehmer $\mathrm{H}$. Development of the CD4 and CD8 lineage of T cells : instruction versus selection. EMBOJ1991; 10 : 913-8.

6. Seong RH, Chamberlain JW, Parnes IR. Signal for T-cell differenciation to a CD4 cell lineage is delivered by $\mathrm{CD} 4$ transmembrane region and/or cytoplasmic tail. Nature $1992 ; 356: 718-20$.

7. Davis CB, Killeen N, Crooks MEC, Raulet D, Littman DR. Evidence for a stochastic mechanism in the differentiation of mature subsets of T-lymphocytes. Cell $1993 ; 73: 237-47$.

8. Itano A, Kioussis D, Robey E. Stochastic component to development of class-I major histocompatibility complex-specific T-cells. Proc Natl Acad Sci USA 1994; 91 : 2204.

9. Chan SH, Cosgrove D, Waltzinger $\mathrm{C}$, Benoist $C$. Mathis D. Another view of the Belective model of thymocyte selection. Cell $1993 ; 73: 22-36$.
10. Bendelac A, Killeen N, Littman DR, Schwartz RH. A subset of CD4(+) thymocytes selected by MHC class molecules. Science $1994 ; 263$ : 1774-8.

11. Huang S, Terstappen LW. Formation of haematopoietic microenvironment and haematopoietic stem cells from single human bone marrow stem cells [see comments] Nature $1992 ; 360: 745-9$.

12. Huang S, Terstappen LWMM. Formation of haematopoietic microenvironment and haematopoietic stem cells from single human bone marrow stem cells. Nature $1994 ; 368: 664$.

13. Baroffio A, Blot M. Statistical evidence for a random commitment of pluripotent cephalic neural crest cells. J Cell Sci 1992 ; $103: 581-7$.

14. Greenwald I, Rubin GM. Making a difference : the role of cell-cell interactions in establishing separate identities for equivalent cells. Cell $1992 ; 68: 271-81$.

15. Ptashne M. A genetic switch : phage l and higher organisms. Cambridge, $\mathrm{MA}$ : Cell Press \& Blackwell Scientific, 1991, $2^{\mathrm{e}}$ ed.

16. Ko MS. Induction mechanism of a single gene molecule: stochastic or deterministic ? Bioessays $1992 ; 14: 341-6$.

17. Ko MS, Nakauchi H, Takahashi N. The dose dependence of glucocorticoidinducible gene expression results from changes in the number of transcriptionally active templates. $E M B O$ J $1990 ; 9: 2835-42$.

18. Ko MS. A stochastic model for gene induction. J Theor Biol $1991 ; 153$ : 181-94.

19. Shea MA, Ackers GK. The OR control system of bacteriophage lambda. A physicalchemical model for gene regulation. J Mol Biol 1985 ; 181 : 211-30.

20. Ackers GK, Johnson AD, Shea MA Quantitative model for gene regulation by 1 phage repressor. Proc Natl Acad Sci USA 1982; $79: 1129-33$

21. Raff MC. Social controls on cell survival and cell death. Nature $1992 ; 356: 397-400$.

\section{Jean Peccoud}

Université Joseph Fourier-Grenoble I, TIMCIMAG URA CNRS D1618, faculté de médecine de Grenoble, $38706 \mathrm{La}$ Tronche Cedex, France.

\section{Stéphane Viville}

Docteur en pharmacie, docteur ès-sciences, post-doctorant, Wellcome/CRC Institut, Tennis Court Road, Cambridge CB2 IQR, Royaume-Uni.

\section{TIRÉS A PART}

\section{J. Peccoud.}

